\title{
LEAST SQUARES APPROXIMATIONS OF POWER SERIES
}

\author{
JAMES GUYKER
}

Received 27 June 2005; Revised 12 June 2006; Accepted 22 June 2006

The classical least squares solutions in $C[-1,1]$ in terms of linear combinations of ultraspherical polynomials are extended in order to estimate power series on $(-1,1)$. Approximate rates of uniform and pointwise convergence are obtained, which correspond to recent results of U. Luther and G. Mastroianni on Fourier projections with respect to Jacobi polynomials.

Copyright (c) 2006 Hindawi Publishing Corporation. All rights reserved.

\section{Introduction}

The ultraspherical or Gegenbauer polynomials $p_{n}(x)$ with given constant $\rho \geq 0$, normalized by $p_{n}(1)=1$, arise in solutions to least squares approximation problems (see $[3,11,12])$ : define an inner product on $C[-1,1]$ by

$$
\langle f, g\rangle:=\int_{-1}^{1} f(x) g(x)\left(1-x^{2}\right)^{(\rho-1) / 2} d x
$$

Then $p_{n}(x)$ is generated by applying the Gram-Schmidt procedure to $1, x, \ldots, x^{n}$, and is given recursively by

$$
\begin{gathered}
p_{0}(x)=1, \quad p_{1}(x)=x, \quad \text { for } n \geq 1, \\
p_{n+1}(x)=-\frac{n}{n+\rho} p_{n-1}(x)+\frac{2 n+\rho}{n+\rho} x p_{n}(x) .
\end{gathered}
$$

For each $f$ in $C[-1,1]$,

$$
\sum_{j=0}^{n}\left\langle f, \frac{p_{j}}{\left\|p_{j}\right\|^{2}}\right\rangle p_{j}(x)
$$

is the unique polynomial which minimizes $\|f-p\|^{2}$ over all polynomials $p$ of degree 
at most $n$. A consequence of a recent result of Luther and Mastroianni [8, Theorem 2.1 (Corollary 3.3)] on Fourier projections with respect to normalized Jacobi polynomials is the following.

If $\rho / 4 \leq \gamma \leq \rho / 4+1 / 2$, then

$$
\left|\left[f(x)-\sum_{j=0}^{n}\left\langle f, \frac{p_{j}}{\left\|p_{j}\right\|^{2}}\right\rangle p_{j}(x)\right]\left(1-x^{2}\right)^{\gamma}\right| \leq c E_{n}^{\gamma}(f) \ln (n+2),
$$

where

$$
E_{n}^{y}(f):=\inf \left\{\left\|[f(x)-p(x)]\left(1-x^{2}\right)^{\gamma}\right\|_{\infty}: p \text { polynomial of degree } \leq n\right\}
$$

and $c$ is independent of $f$ and $n$.

This extends a classical theorem on Chebyshev polynomial $(\rho=0)$ approximation (see [5, Theorem 14.8.2], [11, Theorem 3.3]). In particular, if $n \geq k \geq 1$ and $\| f^{(k)}(x)(1-$ $\left.x^{2}\right)^{\gamma+k / 2} \|_{\infty}<\infty$, then

$$
E_{n}^{\gamma}(f) \leq \frac{c}{(n+1)^{k}} E_{n-k}^{\gamma+k / 2}\left(f^{(k)}\right)
$$

where $c$ is independent of $f$ and $n$, which generalizes Jackson's theorem (see [3, Theorem 4.8], [7], [8, Corollary 3.4]).

In this paper we obtain analogs to (1.4) and (1.6) for power series $f$ defined on the open interval $(-1,1)$. Such functions $f$ (especially without closed forms) arise, for example, in solutions to differential equations. It will be necessary to first extend the above least squares polynomial. This is accomplished in Section 2 by replacing the integral in (1.3) by a sum in terms of Maclaurin coefficients of $f$ and inversion coefficients of expansions of monomials as linear combinations of ultraspherical polynomials. After proving key properties of the latter coefficients in Section 3, we then derive uniform or pointwise estimates to $f$ with these least squares extensions.

\section{Generalized Fourier coefficients}

We first consider a general notion of summability. The following implies the well-known convergence tests of Abel and Dirichlet [2, Theorems 10.17, 10.18] but with modified error estimates.

Proposition 2.1. Suppose that $\sum a_{i}$ and $\sum\left|b_{j+1}-b_{j}\right|$ converge. Then,

$$
\left|\sum_{i>n} a_{i} b_{i}\right| \leq\left(\left|b_{n+1}\right|+\sum_{j>n}\left|b_{j+1}-b_{j}\right|\right) \epsilon_{n}\left(\left\langle a_{i}\right\rangle\right)
$$

where

$$
\epsilon_{n}\left(\left\langle a_{i}\right\rangle\right):=\max \left\{\left|\sum_{i>k} a_{i}\right|: k \geq n\right\}
$$

converges to zero. 
Proof. Note that

$$
\begin{aligned}
\sum_{j>n}\left|\sum_{i>j} a_{i}\right|\left|b_{j+1}-b_{j}\right| & =\lim _{m \rightarrow \infty} \sum_{j=n+1}^{m}\left|\sum_{i>j} a_{i}\right|\left|b_{j+1}-b_{j}\right| \\
& \leq \lim _{m \rightarrow \infty} \epsilon_{n}\left(\left\langle a_{i}\right\rangle\right) \sum_{j=n+1}^{m}\left|b_{j+1}-b_{j}\right|<\infty
\end{aligned}
$$

by the hypotheses. Moreover,

$$
\sum_{j>n}\left|\sum_{i>j} a_{i}\right|\left|b_{j+1}-b_{j}\right|=\lim _{m \rightarrow \infty} \sum_{j=n+1}^{m-1}\left|\sum_{i=j+1}^{m} a_{i}\right|\left|b_{j+1}-b_{j}\right|
$$

since by the triangle inequality,

$$
\begin{aligned}
& \left|\sum_{j>n}\right| \sum_{i>j} a_{i}|| b_{j+1}-b_{j}\left|-\sum_{j=n+1}^{m-1}\right| \sum_{i=j+1}^{m} a_{i}|| b_{j+1}-b_{j}|| \\
& \leq\left|\sum_{i>m} a_{i}\right| \sum_{j=n+1}^{m-1}\left|b_{j+1}-b_{j}\right|+\sum_{j \geq m}\left|\sum_{i>j} a_{i}\right|\left|b_{j+1}-b_{j}\right| \\
& \leq \epsilon_{m}\left(\left\langle a_{i}\right\rangle\right) \sum_{j>n}\left|b_{j+1}-b_{j}\right|
\end{aligned}
$$

which converges to zero as $m$ tends to infinity.

Finally, since

$$
\sum_{i=n+1}^{m} a_{i} b_{i}=\left(\sum_{i=n+1}^{m} a_{i}\right) b_{n+1}+\sum_{j=n+1}^{m-1}\left(\sum_{i=j+1}^{m} a_{i}\right)\left(b_{j+1}-b_{j}\right)
$$

we have

$$
\begin{aligned}
\left|\sum_{i>n} a_{i} b_{i}\right| & \leq\left|\sum_{i>n} a_{i}\right|\left|b_{n+1}\right|+\sum_{j>n}\left|\sum_{i>j} a_{i}\right|\left|b_{j+1}-b_{j}\right| \\
& \leq\left(\left|b_{n+1}\right|+\sum_{j>n}\left|b_{j+1}-b_{j}\right|\right) \epsilon_{n}\left(\left\langle a_{i}\right\rangle\right) .
\end{aligned}
$$

The quantity $\epsilon_{n}\left(\left\langle a_{i}\right\rangle\right)$ was used in [6] to approximate power series with linear combinations of Legendre polynomials $(\rho=1)$. By Abel's theorem [4, page 325], $f(x)=\sum a_{i} x^{i}$ is in $C[-1,1]$ if and only if $\sum a_{2 i}$ and $\sum a_{2 i+1}$ both converge. In this case, we have for $\gamma \geq 0$,

$$
E_{n}^{\gamma}\left(\sum_{i} a_{i} x^{i}\right) \leq 2 \lambda(n+1, \gamma)\left[\epsilon_{n}\left(\left\langle a_{2 i}\right\rangle\right)+\epsilon_{n}\left(\left\langle a_{2 i+1}\right\rangle\right)\right],
$$


4 Least squares approximations of power series

where

$$
\lambda(n, \gamma):=\left(\frac{n}{2 \gamma+n+2}\right)^{(\gamma / 2)(n /(\gamma+1))}\left(\frac{2 \gamma+2}{2 \gamma+n+2}\right)^{\gamma} .
$$

This is immediate from the proposition since for fixed $|x|<1, b_{i}=x^{2 i}\left(1-x^{2}\right)^{\gamma}$ is nonnegative and decreases to zero, and hence

$$
\begin{aligned}
\left|\sum_{i>n} a_{i} x^{i}\left(1-x^{2}\right)^{\gamma}\right| & \leq \sum_{t=0}^{1}|x|^{t}\left|\sum_{2 i+t>n} a_{2 i+t}\left[x^{2 i}\left(1-x^{2}\right)^{\gamma}\right]\right| \\
& \leq \sum_{t=0}^{1} 2|x|^{n+1}\left(1-x^{2}\right)^{\gamma} \epsilon_{n}\left(\left\langle a_{2 i+t}\right\rangle\right) \\
& =2|x|^{(n+1) /(\gamma+1)}\left[|x|^{\gamma((n+1) /(\gamma+1))}\left(1-x^{2}\right)^{\gamma}\right] \sum_{t=0}^{1} \epsilon_{n}\left(\left\langle a_{2 i+t}\right\rangle\right),
\end{aligned}
$$

where $\left\||x|^{\gamma((n+1) /(\gamma+1))}\left(1-x^{2}\right)^{\gamma}\right\|_{\infty}=\lambda(n+1, \gamma)$ by a calculus argument. Note that $\lambda(n, \gamma) \leq$ 1 , and for each $\gamma>0, \lim _{n \rightarrow \infty} \lambda(n, \gamma)=0$; and for each $n>0, \lim _{\gamma \rightarrow \infty} \lambda(n, \gamma)=0$. Furthermore, by Abel's theorem and the proposition, $\left(\sum a_{i} x^{i}\right)^{(k)}$ is in $C[-1,1]$ if and only if the series $\sum a_{2 i+t}(2 i+t)^{k}(t=0,1)$ converge, in which case we have in $(2.10)$ corresponding to $(1.6)$,

$$
\epsilon_{n}\left(\left\langle a_{2 i+t}\right\rangle\right) \leq\left(\frac{2}{n+1}\right)^{k} \epsilon_{n}\left(\left\langle(2 i+t)^{k} a_{2 i+t}\right\rangle\right)
$$

Suppose now that $\sum a_{i} x^{i}$ is a convergent power series on $(-1,1)$ and $p_{n}(x)$ is ultraspherical with constant $\rho \geq 0$. By (1.2), since $x^{i}=x x^{i-1}$, we have the inversion formula

$$
x^{i}=\sum_{j=0}^{i} m_{i j} p_{j}(x)
$$

where $m_{i j}=0$ if $i-j$ is odd, $m_{00}=1$, otherwise

$$
m_{i j}=\frac{j-1+\rho}{2 j-2+\rho} m_{i-1, j-1}+\frac{j+1}{2 j+2+\rho} m_{i-1, j+1}
$$

with $m_{i 1}:=m_{i-1,0}+(1 / 2) m_{i-1,2}$ when $\rho=0$. (We assume $m_{i j}:=0$ if either $i<j$ or $j=-1$.) Clearly $\sum_{j=0}^{i} m_{i j}=1$ and $0<m_{i j} \leq 1$. In fact, (2.13) is equivalent to $m_{11}=1$,

$$
\begin{gathered}
m_{j j}=\frac{j-1+\rho}{2 j-2+\rho} m_{j-1, j-1}, \quad j \geq 2, \\
m_{i+2, j}=\frac{(i+2)(i+1)}{(i-j+2)(i+j+2+\rho)} m_{i j}, \quad i \geq j .
\end{gathered}
$$


This may be verified by first showing by induction on $n$, where $i=j+2 n$ (with $j$ fixed), that if $\left(m_{i j}\right)$ satisfies $(2.14)$, then

$$
m_{i-1, j-1}=\frac{j(2 j-2+\rho)(i+j+\rho)}{i(j-1+\rho)(2 j+\rho)} m_{i j}
$$

with $m_{i-1,0}:=((i+1) / 2 i) m_{i 1}$ when $\rho=0$ and

$$
m_{i-1, j+1}=\frac{(i-j)(j+\rho)(2 j+2+\rho)}{i(j+1)(2 j+\rho)} m_{i j} .
$$

Substituting (2.15) and (2.16) into (2.13), we conclude that the matrices coincide.

By (2.14) we have the following well-known closed form for $m_{i j}$ (see [10, page 283]):

$$
m_{i j}=\frac{(\rho+2 j)((i-j) / 2+1)_{(i-j) / 2}(\rho)_{j}}{2^{i+1}(\rho / 2)_{(i+j+2) / 2}}\left(\begin{array}{l}
i \\
j
\end{array}\right),
$$

where $m_{i j}:=\left(\left(2-\delta_{0 j}\right) / 2^{i}\right)(\underset{(i-j) / 2}{i})$ whenever $\rho=0$. (Recall the factorial function $(\alpha)_{n}:=$ $\alpha(\alpha+1) \cdots(\alpha+n-1)$ when $n \geq 1$ and $(\alpha)_{0}:=1$ for $\alpha \neq 0$.)

We now define the general Fourier coefficient $c_{j}$ of $\sum a_{i} x^{i}$ with respect to the sequence $\left\langle p_{n}\right\rangle$ by

$$
c_{j}:=\sum_{i} a_{i} m_{i j}
$$

whenever this sum converges. Note that $c_{n j}:=\sum_{i=0}^{n} a_{i} m_{i j}$ is the $j$ th coefficient in the expansion of the partial sum

$$
\sum_{i=0}^{n} a_{i} x^{i}=\sum_{i=0}^{n} a_{i}\left(\sum_{j=0}^{i} m_{i j} p_{j}(x)\right)=\sum_{j=0}^{n} c_{n j} p_{j}(x) .
$$

If $c_{j}$ exists for every $j$, then for $\gamma \geq 0$ we also have

$$
\begin{aligned}
\left|\left[\sum a_{i} x^{i}-\sum_{j=0}^{n} c_{j} p_{j}(x)\right]\left(1-x^{2}\right)^{\gamma}\right| & =\left|\sum_{i>n} a_{i} x^{i}\left(1-x^{2}\right)^{\gamma}+\sum_{j=0}^{n}\left(c_{n j}-c_{j}\right) p_{j}(x)\left(1-x^{2}\right)^{\gamma}\right| \\
& \leq\left|\sum_{i>n} a_{i} x^{i}\left(1-x^{2}\right)^{\gamma}\right|+\sum_{j=0}^{n}\left|\sum_{i>n} a_{i} m_{i j}\right| \mu(j, \gamma),
\end{aligned}
$$

where $\mu(j, \gamma):=\left\|p_{j}(x)\left(1-x^{2}\right)^{\gamma}\right\|_{\infty}$. Note that $p_{j}(x)$ is bounded by one in $[-1,1]$ since $p_{j}(1)=1$, and hence by [12, page 95], $p_{j}$ is a convex combination of Chebyshev polynomials. Thus $\mu(j, \gamma) \leq 1$. Moreover, $\mu(0, \gamma)=\mu(j, 0)=1$; and for $j \gamma \neq 0$, since $p_{j}(-x)=$ $(-1)^{j} p_{j}(x)$ and

$$
\left\|x^{j}\left(1-x^{2}\right)^{\gamma}\right\|_{\infty}=\left(\frac{j}{2 \gamma+j}\right)^{j / 2}\left(\frac{2 \gamma}{2 \gamma+j}\right)^{\gamma}
$$


6 Least squares approximations of power series

as above, it follows that

$$
\mu(2 j-t, \gamma) \geq\left|p_{2 j-t}(0)\right|=(1-t) \prod_{k=1}^{j} \frac{2 k-1}{2 k-1+\rho}
$$

and $\lim _{\gamma \rightarrow \infty} \mu(2 j-t, \gamma)=\left|p_{2 j-t}(0)\right|$. Furthermore we have the following.

LEMmA 2.2. If $0<\rho / 4 \leq \gamma$, then there exists a constant $c=c(\rho, \gamma)$ independent of $j$ such that

$$
\mu(j, \gamma)^{2} \leq c \frac{j !}{(\rho)_{j}(2 j+\rho)}, \quad j=0,1, \ldots
$$

Proof. Suppose that $\rho / 4 \leq \gamma$. By $[8$, Remark 2.4],

$$
\sup _{j}\left\|\frac{p_{j}(x)}{\left\|p_{j}\right\|}\left(1-x^{2}\right)^{\gamma}\right\|_{\infty}^{2}<\infty .
$$

Since $p_{j}(1)=1$ and the orthonormal sequence $\left\{p_{j} /\left\|p_{j}\right\|\right\}$ is unique (see $[3$, Theorem 4.2]), it follows from [12, page 82, equation 4.7.15] that for $\rho>0$,

$$
\left\|p_{j}\right\|^{2}=\frac{2^{2-\rho} \pi \Gamma(\rho) j !}{\Gamma(\rho / 2)^{2}(\rho)_{j}(2 j+\rho)} .
$$

(Incidentally, if $\rho=0$ then $\left\|p_{0}\right\|^{2}=\pi$ and $\left\|p_{j}\right\|^{2}=\pi / 2$ for $j>0$.)

\section{Main properties of inversion coefficients}

We will use (2.20) to approximate series $\sum a_{i} x^{i}$ on $(-1,1)$ such that $\sum\left(a_{i} / i^{s}\right)$ converges for some nonnegative number $s$. Since $c_{j}=\sum_{i}\left(a_{i} / i^{s}\right)\left(i^{s} m_{i j}\right)$, we first investigate convergence of the sequence $i^{s} m_{i j}$ for fixed $j$. We begin with the following technical result.

Lemma 3.1. Let $s \geq-1$ be real, and $N:=2(1+s) /-s$ if $-1<s<0$ and $N:=1$ otherwise. The function

$$
h_{s}(x):=(x+1)\left(\frac{x+2}{x}\right)^{s}-x
$$

is monotonically decreasing on $[N, \infty)$ with limit $2 s+1$ as $x$ approaches $\infty$.

Proof. Letting $y=2 / x$, we have that $h_{s}^{\prime}(x) \leq 0$ for $x \geq N$ if and only if

$$
f_{s}(y):=(1+y)^{1-s}+\frac{s}{2} y^{2}-(1-s) y-1 \geq 0
$$

for $0<y \leq 2 / N$. Now $f_{s}(0)=0$, and $f_{s}^{\prime}(y) \geq 0$ is equivalent to

$$
g_{s}(y):=(1+y)^{s}[1-s(1+y)] \leq 1-s .
$$

However $g_{s}(0)=1-s$ and

$$
g_{s}^{\prime}(y)=(-s)(1+y)^{s-1}[s+(1+s) y] \leq 0
$$

on $(0,2 / N]$. Hence $h_{s}(x)$ is decreasing on $[N, \infty)$. 
Finally note that

$$
h_{s}(x)=\left(1+\frac{2}{x}\right)^{s}+\frac{(1+2 / x)^{s}-1}{1 / x}
$$

and hence the limit follows from L'Hôpital's rule.

The next result is the key to our approximations.

Theorem 3.2. Let $p_{n}(x)$ be ultraspherical with constant $\rho \geq 0$, let $m_{i j}$ be defined by (2.13), and suppose that $s \geq 0$. Then for each $j$,

$$
L(j):=\lim _{n \rightarrow \infty}(j+2 n)^{s} m_{j+2 n, j}
$$

exists if and only if $\rho \geq 2 s-1$. If $\rho \leq 2 s-1$, then the sequence $(j+2 n)^{s} m_{j+2 n, j}$ is monotonically increasing. If $\rho=2 s-1$, then with $s^{\prime}:=-\lfloor-s\rfloor(\lfloor\cdot\rfloor=$ greatest integer function) and $\rho^{\prime}:=-\lfloor-\rho\rfloor$,

$$
\begin{aligned}
L(j) & \leq \frac{2 j+\rho}{j !}(1+\rho)(2+\rho) \cdots(j-1+\rho)\left[1 \cdot 3 \cdot 5 \cdots\left(2 s^{\prime}-1\right)\right] \\
& \leq \frac{2 j+\rho^{\prime}}{\rho^{\prime} !}(j+1)(j+2) \cdots\left(j+\rho^{\prime}-1\right)\left[1 \cdot 3 \cdot 5 \cdots\left(2 s^{\prime}-1\right)\right], \quad \rho \neq 0,
\end{aligned}
$$

where the inequalities are equalities if $s$ is an integer; and the equality holds in the latter inequality if $\rho$ is an integer.

On the other hand suppose that $\rho>2 s-1$. Then for each $j$,

(a) $L(j)=0$,

(b) the sequence $(j+2 n)^{s-1} m_{j+2 n, j}$ is summable,

(c) there exists an integer $I(j) \geq j$ such that whenever $i-j$ is even the following are equivalent:

(i) $(i+2)^{s} m_{i+2, j} \leq i^{s} m_{i j}$;

(ii) $i \geq I(j)$;

(iii) $i^{s} j(j+\rho) \leq(i+2)\left\{i^{s}(\rho-1)-2 i^{s-1}-(i+1)(i+2)\left[(i+2)^{s-1}-i^{s-1}\right]\right\}$, and

(d) $i^{s} m_{i j}=O\left(i^{-[\rho-(2 s-1)-r] / 2}\right)$ for any $r$ in $(0, \rho-(2 s-1))$.

Proof. Let $s \geq 0$ and $0 \neq i \geq j$. Then by (2.14), we have

$$
(i+2)^{s} m_{i+2, j}-i^{s} m_{i j}=\frac{A_{i}}{(1-j /(i+2))(1+(j+2+\rho) / i)} i^{s-1} m_{i j}
$$

where $A_{i}=j(j+\rho) /(i+2)+h_{s}(i)-(\rho+2)$ with $h_{s}(x)$ given by Lemma 3.1. It follows that $\left\langle(j+2 n)^{s} m_{j+2 n, j}\right\rangle$ is Cauchy if and only if

$$
\sum_{i} \frac{A_{i}\left(i^{s-1} m_{i j}\right)}{(1-j /(i+2))(1+(j+2+\rho) / i)}<\infty .
$$

Now

$$
0<\left(1-\frac{j}{j+2}\right) \leq\left(1-\frac{j}{i+2}\right)\left(1+\frac{j+2+\rho}{i}\right) \leq 3+j+\rho
$$


and $A_{i}$ decreases to $2 s-1-\rho$. Hence if $\left\langle(j+2 n)^{s-1} m_{j+2 n, j}\right\rangle$ is summable, then $L(j)$ exists by Proposition 2.1. Similarly, the converse is true if $\rho \neq 2 s-1$ since $A_{i}^{-1}$ increases monotonically to $(2 s-1-\rho)^{-1}$.

As in (3.8), we have

$$
i\left[\frac{i^{s-1} m_{i j}}{(i+2)^{s-1} m_{i+2, j}}-1\right]=\frac{(1+2 / i)\left[\rho+2-h_{s-1}(i)\right]-j(j+\rho) / i}{(1+2 / i)^{s}(1+1 / i)}
$$

which converges to $\rho-2 s+3$ by Lemma 3.1. Thus letting $i=j+2 n$ we have

$$
\rho-2 s+3=j(0)+2 \lim _{n \rightarrow \infty}\left[n \frac{(j+2 n)^{s-1} m_{j+2 n, j}}{(j+2 n+2)^{s-1} m_{j+2 n+2, j}}-1\right] .
$$

By Raabe's test [9, page 396], $\sum_{i} i^{s-1} m_{i j}$ converges when the limit in this identity is greater than one and diverges when it is less than one. Hence $L(j)$ exists (and (b) follows) if $\rho>2 s-1$, and fails to exist if $\rho<2 s-1$.

Next suppose that $\rho>2 s-1$. We show $L(j)=0$ by verifying $\sum_{i}\left(i^{s} m_{i j}\right)^{p}<\infty$ for some positive constant $p$. By Raabe's test as above,

$$
i\left\{\left[\frac{i^{s} m_{i j}}{(i+2)^{s} m_{i+2, j}}\right]^{p}-1\right\}=(i X) \frac{(1+X)^{p}-1}{X},
$$

where

$$
X=\frac{\rho+2-h_{s}(i)-j(j+\rho) /(i+2)}{(i+1)(1+2 / i)^{s}} .
$$

Hence

$$
\lim _{i \rightarrow \infty} X=0, \quad \lim _{i \rightarrow \infty} i X=\rho-(2 s-1) .
$$

Therefore with $i=j+2 n$,

$$
p[\rho-(2 s-1)]=j(0)+2 \lim _{n \rightarrow \infty} n\left\{\left[\frac{(j+2 n)^{s} m_{j+2 n, j}}{(j+2 n+2)^{s} m_{j+2 n+2, j}}\right]^{p}-1\right\} .
$$

By Raabe's test $\sum_{i}\left(i^{s} m_{i j}\right)^{p}<\infty$ if $p$ is chosen such that $p[\rho-(2 s-1)]>2$. Thus $L(j)=0$.

By (3.8), $(i+2)^{s} m_{i+2, j} \leq i^{s} m_{i j}$ if and only if

$$
\frac{j(j+\rho)}{i+2}+h_{s}(i) \leq \rho+2,
$$

where $h_{s}(i), i \geq 1$, decreases to $2 s+1$ by Lemma 3.1. If $\rho \leq 2 s-1$, then

$$
\rho+2 \leq 2 s+1 \leq h_{s}(i) \leq \frac{j(j+\rho)}{i+2}+h_{s}(i)
$$

so $\left\langle(j+2 n)^{s} m_{j+2 n, j}\right\rangle$ is increasing. 
Finally if $\rho>2 s-1$ (and thus $2 s+1<\rho+2$ ), then there exists an integer $I(j) \geq j$ such that (3.17) holds if and only if $i \geq I(j)$. Furthermore, (c)(iii) follows from (3.17) and the identity

$$
h_{s-1}(x)=3+\frac{2}{x}+\left(\frac{x+1}{x}\right)\left(\frac{x+2}{x}\right)\left[\frac{(x+2)^{s-2}-x^{s-2}}{x^{s-3}}\right] .
$$

In order to show (d), let $b_{n}:=\left[(j+2 n)^{s} m_{j+2 n, j}\right]^{p}$, where $p$ is chosen above. Let $q$ be in the interval $(1, p[\rho-(2 s-1)] / 2)$. There exists an integer $N_{0}$ such that if $n \geq N_{0}$, then $q b_{n+1}<n\left(b_{n}-b_{n+1}\right)$. Thus if $N=\max \left\{I(j), N_{0}\right\}$, then

$$
q \sum_{i=1}^{m} b_{N+i}<N b_{N}+\sum_{i=1}^{m-1} b_{N+i}-(N+m-1) b_{N+m}
$$

so by (c)(i) it follows that

$$
(q-1) m b_{N+m} \leq(q-1) \sum_{i=1}^{m} b_{N+i}<N b_{N}-(N+m) b_{N+m} .
$$

Therefore for every $m=1,2, \ldots$,

$$
b_{N+m}<\frac{N b_{N}}{N+q m}<\frac{N b_{N}}{N+m}
$$

and if $r:=\rho-(2 s-1)-2 / p$, then $r$ satisfies (d).

Finally suppose that $\rho=2 s-1$. By solving (2.15) for $m_{i j}$ when $i-j$ is even, we have

$$
i^{s} m_{i j}=\frac{2 j+\rho}{j !}(1+\rho)(2+\rho) \cdots(j-1+\rho)\left(\prod_{k=0}^{j-1} \frac{i-k}{i+j+\rho-2 k}\right)\left(i^{s} m_{i-j, 0}\right),
$$

where $m_{i-j, 0}=1 \cdot 3 \cdot 5 \cdots(i-j-1) /(2+\rho)(4+\rho) \cdots(i-j+\rho)$. Note that if $s$ is a positive integer, then

$$
m_{i-j, 0}=\frac{1 \cdot 3 \cdot 5 \cdots(2 s-1)}{(i-j+1)(i-j+3) \cdots(i-j+2 s-1)}
$$

and hence $L(j)$ is given with $s^{\prime}=s$ and equality in both inequalities in (3.7). Assume that $s$ is not an integer and $i-j=2 n$. Since $i^{s} m_{i j}$ is increasing and $i^{s} m_{i j} / i^{s} m_{i-j, 0}$ is bounded by and converges to $((2 j+\rho) / j !)(1+\rho) \cdots(j-1+\rho)$ by $(3.23)$, it suffices to show that $(j+2 n)^{s} m_{2 n, 0}$ converges and is bounded by $1 \cdot 3 \cdots\left(2 s^{\prime}-1\right)$. This will follow from the integer case once we show for fixed $j$ and $n$ that

$$
f(s):=\frac{(j+2 n)^{s}}{(2 s+1)(2 s+3) \cdots(2 s+2 n-1)}
$$

is monotonically increasing for $s \geq 1 / 2$ since $\rho=2 s-1 \geq 0$. But $f^{\prime}(s) \geq 0$ if and only if

$$
\ln (j+2 n) \geq 2\left(\frac{1}{2 s+1}+\frac{1}{2 s+3}+\cdots+\frac{1}{2 s+2 n-1}\right) .
$$


Since the right side is decreasing and $\ln (j+2 n) \geq \ln (2 n)$, the inequality will follow if we show $\ln (2 n) \geq \sum_{k=1}^{n}(1 / k)$. Now for every integer $m$ in $(1, n)$,

$$
\begin{aligned}
\ln (n) & =\int_{1}^{n} \frac{d x}{x} \geq \ln (m)+\sum_{k=m+1}^{n} \frac{1}{k} \geq \ln (m-1)+\sum_{k=m}^{n} \frac{1}{k} \\
& \geq \ln (1)+\sum_{k=2}^{n} \frac{1}{k}=\sum_{k=1}^{n} \frac{1}{k}-1 .
\end{aligned}
$$

Thus,

$$
\sum_{k=1}^{m-1} \frac{1}{k}-\ln (m-1)+\ln (n) \geq \sum_{k=1}^{m} \frac{1}{k}-\ln (m)+\ln (n) \geq \sum_{k=1}^{n} \frac{1}{k} .
$$

Since $\sum_{k=1}^{4}(1 / k)-\ln (4)<\ln (2)<\sum_{k=1}^{5}(1 / k)-\ln (5)$, we have that $\ln (2)+\ln (n) \geq \sum_{k=1}^{n}(1 / k)$ for $n \geq 5$.

Hence for fixed $n \geq 5, f(s)$ is increasing and $f(s) \leq f\left(s^{\prime}\right)$. Therefore $(j+2 n)^{s} m_{2 n, 0}$ is bounded and thus $i^{s} m_{i j}$ converges. It follows that

$$
(j+2 n)^{s} m_{2 n, 0}=\frac{i^{s} m_{i j}}{\left(i^{s} m_{i j}\right) /\left(i^{s} m_{i-j, 0}\right)}
$$

converges and has limit bounded by $1 \cdot 3 \cdots\left(2 s^{\prime}-1\right)$. Finally $\rho \leq \rho^{\prime}$ and the second inequality follows by multiple cancellations when $\rho \neq 0$.

If $s$ is an integer, then $I(j)$ may be estimated easily.

Proposition 3.3. Let $s$ be a nonnegative integer, $\rho>2 s-1$, and let $I(j)$ be given as in Theorem 3.2. Consider the following expressions:

$$
\begin{gathered}
\sigma_{k}=2^{s+1-k}\left[\left(\begin{array}{c}
s+1 \\
s+2-k
\end{array}\right)+\left(\begin{array}{c}
s+2 \\
s+2-k
\end{array}\right)\right], k=0,1, \ldots, s-2, \\
\sigma_{s-1}=4\left[s^{2}+\left(\begin{array}{c}
s \\
3
\end{array}\right)+\left(\begin{array}{c}
s+1 \\
3
\end{array}\right)\right], \\
\sigma_{s}=\sigma_{s}(j)=j(j+\rho)-2\left(\rho-s^{2}-2 s+1\right), \\
\sigma_{s+1}=\rho-(2 s-1) .
\end{gathered}
$$

If $s=0$, then $I(0)=0$ and $I(j)=-\left\lfloor-\sigma_{s} / \sigma_{s+1}\right\rfloor$ when $j \geq 1$; and if $s=1$, then $I(0)=$ $-\left\lfloor-\sigma_{s-1} / 2 \sigma_{s+1}\right\rfloor$. Otherwise, let $s=3 k^{\prime}+t^{\prime}$ for nonnegative integers $k^{\prime}$ and $t^{\prime} \leq 2$, and define

$$
R(j):=1+\frac{\max \left\{\sigma_{s}(j), \sigma_{k^{\prime}+1}\right\}}{\sigma_{s+1}} .
$$

Then $I(j)<R(j)+1$, and if $\sigma_{s}(j) \geq \sigma_{k^{\prime}+1}$, then $R(j)-1<I(j)$. In particular, if $\rho=2 s$, then $I(j)=R(j)$. 
Proof. By the binomial theorem and the identity $\left(\begin{array}{c}s \\ k\end{array}\right)+\left(\begin{array}{c}s \\ k+1\end{array}\right)=\left(\begin{array}{c}s+1 \\ k+1\end{array}\right)$, a straightforward computation shows that (c)(iii) of Theorem 3.2 is equivalent to

$$
\sigma_{s+1} i^{s+1} \geq \sum_{k=0}^{s} \sigma_{k} i^{k} .
$$

The special cases $s=0$ and $s=1$, when $j=0$, follow easily from (3.32). Thus let $s=$ $3 k^{\prime}+t^{\prime}$ in the other cases. By the following argument (which is usually given to bound the zeros of a polynomial [1, Theorem 6.1$]$ ) we have if

$$
i \geq M(j):=\max \left\{\frac{\sigma_{0}}{\sigma_{s+1}}, 1+\frac{\sigma_{k}}{\sigma_{s+1}}: k=1, \ldots, s\right\},
$$

then $i$ satisfies (3.32),

$$
\begin{aligned}
\sum_{k=0}^{s} \sigma_{k} i^{k} & \leq\left[\sigma_{s+1}+\sigma_{s+1}(i-1)\right]+\sigma_{s+1}(i-1) \sum_{k=1}^{s} i^{k} \\
& =\sigma_{s+1}\left[1+(i-1) \frac{i^{s+1}-1}{i-1}\right]=\sigma_{s+1} i^{s+1} .
\end{aligned}
$$

Thus $I(j)<M(j)+1$.

We will show that $M(j)=R(j)$ for all $j$, which will imply the proposition since in the case $\sigma_{s}(j) \geq \sigma_{k^{\prime}+1}$ we will then have that $M(j)=1+\sigma_{s}(j) / \sigma_{s+1}$ and $x=M-1$ fails to satisfy (3.32). If

$$
\sigma_{s}(M-1)^{s}=\sigma_{s+1} x^{s+1} \geq \sum_{k=0}^{s} \sigma_{k} x^{k},
$$

then $0 \geq \sum_{k=0}^{s-1} \sigma_{k}(M-1)^{k}$ which is impossible since $M \geq 1, \sigma_{k} \geq 0$, and $\sigma_{0}=2^{s+1}>0$. Thus by (3.17), since $h_{s}(x)$ decreases on $[1, \infty)$, it will follow that $M(j)-1<I(j)$.

Thus it remains to show $M(j)=R(j)$. The following results may be readily established from the given definitions:

(a) if $s=1(j \neq 0), 2$, or 3 , then $M(j)=R(j)$;

(b) $\sigma_{0} / \sigma_{s+1} \leq 1+\sigma_{1} / \sigma_{s+1}$ for all $s \geq 2$.

We therefore assume henceforth that $s=3 k^{\prime}+t^{\prime} \geq 4$, where $k^{\prime} \geq 1$ and $t^{\prime}=0,1$, or 2 , and we seek to verify that

$$
\max \left\{\sigma_{1}, \ldots, \sigma_{s}\right\}=\max \left\{\sigma_{s}, \sigma_{k^{\prime}+1}\right\} .
$$

We first show that $\sigma_{s-1}<\sigma_{s-2}$ which may be rewritten as

$$
s^{2}<\left(\begin{array}{c}
s+2 \\
4
\end{array}\right)+2\left(\begin{array}{c}
s+1 \\
4
\end{array}\right)+\left(\begin{array}{l}
s \\
4
\end{array}\right)
$$

or upon further simplification as

$$
\frac{24 s}{s-1}<(s+2)(s+1)+2(s-2)(s+1)+(s-2)(s-3) .
$$


This inequality is true when $s=4$, and for $s \geq 4$ the left side decreases and the right side increases. Hence $\sigma_{s-1}<\sigma_{s-2}$.

Next we prove that

$$
\text { for } i=1, \ldots, s-3, \quad \text { it follows that } \sigma_{i+1}>\sigma_{i} \text { iff } s \geq 3 i \text {. }
$$

As above, we have that $\sigma_{i+1}>\sigma_{i}$ is equivalent to

$$
\left(\begin{array}{c}
s+2 \\
s+1-i
\end{array}\right)>3\left(\begin{array}{c}
s+1 \\
s+2-i
\end{array}\right)+\left(\begin{array}{c}
s+2 \\
s+2-i
\end{array}\right)
$$

which in turn is equivalent to

$$
(3 i-s-1)(i+s+2)+2 i<0, \quad i=1, \ldots, s-3 .
$$

If $s \geq 3 i$, then $(3 i-s-1)(i+s+2)+2 i \leq i-s-2 \leq-5$ since $i \leq s-3$. And if $s<3 i$, then $s=3 i-k$ for some integer $k \geq 1$. In this case

$$
(3 i-s-1)(i+s+2)+2 i=(k-1)(i+s+2)+2 i \geq 0 .
$$

Hence (3.39) follows.

Finally since $s=3 k^{\prime}+t^{\prime} \geq 3 i$ for $i=1, \ldots, k^{\prime}$, we have from (3.39) that $\sigma_{k+1}>\sigma_{k}(k=$ $\left.1, \ldots, k^{\prime}\right)$. Moreover, since $s<3 i$ for $i=k^{\prime}+1, \ldots, s-3$, it follows from (3.39) that $\sigma_{k^{\prime}+k} \geq$ $\sigma_{k^{\prime}+k+1}\left(k=1, \ldots, s-3-k^{\prime}\right)$. Since $\sigma_{s-1}<\sigma_{s-2}$ above, we have the desired identity (3.36).

Remark 3.4. For each $s$ in Proposition 3.3, there exists $J=J(s)$ such that

$$
R(j)-1<I(j)<R(j)+1
$$

for all $j \geq J$.

\section{Approximating power series}

We consider power series $\sum a_{i} x^{i}$ such that the series $\sum a_{2 i+t} \beta_{2 i+t}(t=0,1)$ converge for some positive sequences $\beta_{2 i+t}$ that are either both monotonically increasing or both satisfy $\lim _{i \rightarrow \infty}\left(\beta_{2(i+1)+t} / \beta_{2 i+t}\right)=1$. If they are both increasing, then by Proposition 2.1 and the ratio test, $\sum a_{i} x^{i}$ converges uniformly on $[-1,1]$, and as in $(2.10)$ we have the estimates for $\gamma \geq 0$ :

$$
\begin{aligned}
\left|\sum_{i>n} a_{i} x^{i}\left(1-x^{2}\right)^{\gamma}\right| & \leq \sum_{t=0}^{1}|x|^{t}\left|\sum_{2 i+t>n}\left(a_{2 i+t} \beta_{2 i+t}\right)\left[\frac{x^{2 i}\left(1-x^{2}\right)^{\gamma}}{\beta_{2 i+t}}\right]\right| \\
& \leq \sum_{t=0}^{1} \frac{2|x|^{n+1}\left(1-x^{2}\right)^{\gamma}}{\beta_{n+1}} \epsilon_{n}\left(\left\langle a_{2 i+t} \beta_{2 i+t}\right\rangle\right) \\
& \leq 2|x|^{(n+1) /(\gamma+1)} \frac{\lambda(n+1, \gamma)}{\beta_{n+1}} \sum_{t=0}^{1} \epsilon_{n}\left(\left\langle a_{2 i+t} \beta_{2 i+t}\right\rangle\right) .
\end{aligned}
$$


On the other hand, if the sequences satisfy $\lim _{i \rightarrow \infty}\left(\beta_{2(i+1)+t} / \beta_{2 i+t}\right)=1$, then $\sum a_{i} x^{i}$ converges pointwise on $(-1,1)$ and for each $x$ in $(-1,1)$, there exists $N=N(x)$ such that inequalities (4.1) hold for $n \geq N$.

We begin with the uniformly convergent case.

Theorem 4.1. Suppose that $\sum a_{i} x^{i}$ is a power series such that the series $\sum a_{2 i+t} \beta_{2 i+t}(t=0,1)$ converge for some positive, monotonically increasing sequences $\beta_{2 i+t}$, let $\gamma \geq 0$, and let $p_{n}(x)$ be ultraspherical with constant $\rho \geq 0$. For every $j$ and $t$, the general Fourier coefficient $c_{2 j+t}$ of $\sum a_{i} x^{i}$ with respect to $\left\langle p_{n}\right\rangle$ exists and satisfies

$$
\left|c_{2 j+t}-\sum_{i=0}^{n} a_{i} m_{i, 2 j+t}\right| \leq 2\left(\frac{2}{\beta_{n+1}}-\lim _{i \rightarrow \infty} \frac{1}{\beta_{2 i+t}}\right) m_{\max \{I(2 j+t), n+1\}, 2 j+t} \epsilon_{n}\left(\left\langle a_{2 i+t} \beta_{2 i+t}\right\rangle\right),
$$

where $m_{\max \{I(2 j+t), n+1\}, 2 j+t}=O\left((n+1)^{-(\rho+1-r) / 2}\right) \leq 1$ for any $r$ in $(0, \rho+1)$.

Moreover, for each $x$ in $[-1,1]$,

$$
\begin{aligned}
& \left|\left[\sum a_{i} x^{i}-\sum_{j=0}^{n} c_{j} p_{j}(x)\right]\left(1-x^{2}\right)^{\gamma}\right| \\
& \leq \sum_{t=0}^{1} 2\left\{|x|^{(n+1) /(\gamma+1) \frac{\lambda(n+1, \gamma)}{\beta_{n+1}}}\right. \\
& \qquad\left(\frac{2}{\beta_{n+1}}-\lim _{i \rightarrow \infty} \frac{1}{\beta_{2 i+t}}\right)\left[\sum_{2 j+t \leq \rho_{n}} m_{n+1,2 j+t} \mu(2 j+t, \gamma)\right. \\
& \left.\left.\quad+\sum_{\rho_{n}<2 j+t \leq n} m_{I(2 j+t), 2 j+t} \mu(2 j+t, \gamma)\right]\right\} \epsilon_{n}\left(\left\langle a_{2 i+t} \beta_{2 i+t}\right\rangle\right),
\end{aligned}
$$

where

$$
\begin{gathered}
\rho_{n}:=\frac{1}{2}\left(\sqrt{\rho^{2}+4(n-2)(\rho+1)}-\rho\right), \\
\sum_{2 j+t \leq \rho_{n}} m_{n+1,2 j+t} \mu(2 j+t, \gamma) \leq 1 .
\end{gathered}
$$

If $1 / 4 \leq \rho / 4 \leq \gamma$, then there exists a constant $c$ independent of $n$ such that for $n \geq 2$,

$$
\begin{aligned}
& \sum_{\rho_{n}<2 j+t \leq n} m_{I(2 j+t), 2 j+t} \mu(2 j+t, \gamma) \\
& \quad \leq c\left[\frac{1}{\sqrt{2 t+\rho}}+\frac{1}{\sqrt{4+2 t+\rho}}+\frac{1}{2}\left(\sqrt{4\left\lfloor\frac{n-t}{2}\right\rfloor+2 t+\rho}-\sqrt{4+2 t+\rho}\right)\right]
\end{aligned}
$$


14 Least squares approximations of power series

and hence convergence is uniform in (4.3) if

$$
\lim _{n \rightarrow \infty} \frac{\sqrt{n}}{\beta_{n+1}} \epsilon_{n}\left(\left\langle a_{2 i+t} \beta_{2 i+t}\right\rangle\right)=0, \quad t=0,1 .
$$

Proof. By Theorem 3.2 with $s=0$ and Proposition 2.1, we have that

$$
\left|c_{2 j+t}-\sum_{i=0}^{n} a_{i} m_{i, 2 j+t}\right|=\left|\sum_{2 i+t>n} a_{2 i+t} m_{2 i+t, 2 j+t}\right| \leq 2 m_{\max \{I(2 j+t), n+1\}, 2 j+t} \epsilon_{n}\left(\left\langle a_{2 i+t}\right\rangle\right),
$$

where $m_{\max \{I(2 j+t), n+1\}, 2 j+t}$ satisfies the given identity,

$$
\epsilon_{n}\left(\left\langle a_{2 i+t}\right\rangle\right)=\max \left\{\left|\sum_{2 i+t>k}\left(a_{2 i+t} \beta_{2 i+t}\right)\left(\frac{1}{\beta_{2 i+t}}\right)\right|: k \geq n\right\},
$$

and $1 / \beta_{2 i+t}$ decreases with nonnegative limit. Thus by Proposition 2.1,

$$
\begin{aligned}
\epsilon_{n}\left(\left\langle a_{2 i+t}\right\rangle\right) & \leq \max \left\{\epsilon_{k}\left(\left\langle a_{2 i+t} \beta_{2 i+t}\right\rangle\right)\left(\frac{2}{\beta_{k+1}}-\lim _{i \rightarrow \infty} \frac{1}{\beta_{2 i+t}}\right): k \geq n\right\} \\
& \leq\left(\frac{2}{\beta_{n+1}}-\lim _{i \rightarrow \infty} \frac{1}{\beta_{2 i+t}}\right) \epsilon_{n}\left(\left\langle a_{2 i+t} \beta_{2 i+t}\right\rangle\right) .
\end{aligned}
$$

Therefore (4.2) follows from (4.7).

By (2.20), we have

$$
\begin{aligned}
& \left|\left[\sum a_{i} x^{i}-\sum_{j=0}^{n} c_{j} p_{j}(x)\right]\left(1-x^{2}\right)^{\gamma}\right| \\
& \quad \leq\left|\sum_{i>n} a_{i} x^{i}\left(1-x^{2}\right)^{\gamma}\right|+\sum_{t=0}^{1} \sum_{2 j+t \leq n}\left|\sum_{2 i+t>n} a_{2 i+t} m_{2 i+t, 2 j+t}\right| \mu(2 j+t, \gamma) .
\end{aligned}
$$

Hence (4.3) follows from (4.1) and (4.2) where, by Proposition 3.3, $I(j)=(j(j+\rho)-$ $2(\rho+1)) /(\rho+1)$, and therefore $I(2 j+t) \leq n+1$ if and only if $2 j+t \leq \rho_{n}$.

Suppose that $1 / 4 \leq \rho / 4 \leq \gamma$. By Lemma $2.2, \mu(j, \gamma)^{2} \leq c /(2 j+\rho)$ for all $j$, and $m_{I(2 j+t), 2 j+t} \leq 1$, so the estimate follows by the proof of the integral test since for $m \geq 2$,

$$
\sum_{j=0}^{m} \frac{1}{\sqrt{2(2 j+t)+\rho}} \leq \frac{1}{\sqrt{2 t+\rho}}+\frac{1}{\sqrt{2(2+t)+\rho}}+\frac{1}{2}(\sqrt{2(2 m+t)+\rho}-\sqrt{2(2+t)+\rho}) .
$$

Remark 4.2. If

$$
\lim _{n \rightarrow \infty} \frac{n}{\beta_{n+1}} \epsilon_{n}\left(\left\langle a_{2 i+t} \beta_{2 i+t}\right\rangle\right)=0, \quad t=0,1,
$$

then convergence in $(4.3)$ is uniform since $m_{i j} \mu(j, \gamma) \leq 1$ for all $i$ and $j$. 
The quantity $\epsilon_{n}\left(\left\langle a_{i}\right\rangle\right)$ was approximated in [6] for the standard Maclaurin series of calculus. We will illustrate the other parts of our estimates. For Legendre polynomials, we have an estimate that is comparable to (1.4).

Example 4.3. Let $\rho=1$ in Theorem 4.1. By [6], $m_{i j} \leq 2 /(j+1)$ for all $i$ and by the proof of the integral test,

$$
\sum_{j=0}^{m} \frac{2}{2 j+t+1} \leq 2-t+\ln [(2-t) m+1]
$$

Hence in (4.3), it follows that

$$
\sum_{\rho_{n}<2 j+t \leq n} m_{I(2 j+t), 2 j+t} \mu(2 j+t, \gamma) \leq \max _{\rho_{n}<2 j+t \leq n} \mu(2 j+t, \gamma)\left\{2-t+\ln \left[(2-t)\left\lfloor\frac{n-t}{2}\right\rfloor+1\right]\right\} .
$$

Since

$$
\lim _{n \rightarrow \infty} \frac{2-t+\ln [(2-t)\lfloor(n-t) / 2\rfloor+1]}{\ln (n+2)}=1,
$$

we have as with (1.4), if

$$
\lim _{n \rightarrow \infty} \max _{\rho_{n}<2 j+t \leq n} \mu(2 j+t, \gamma) \epsilon_{n}\left(\left\langle a_{2 i+t} \beta_{2 i+t}\right\rangle\right) \frac{\ln (n+2)}{\beta_{n+1}}=0, \quad t=0,1,
$$

then the approximations in (4.3) converge uniformly.

However, if $1 / 4=\rho / 4 \leq \gamma$, then by Lemma 2.2

$$
m_{I(2 j+t), 2 j+t} \mu(2 j+t, \gamma) \leq c \frac{2}{2 j+t+1} \frac{1}{\sqrt{2(2 j+t)+1}}
$$

and for $m \geq 2$,

$$
\sum_{j=0}^{m} \frac{1}{(2 j+t+1)^{3 / 2}} \leq 2^{-(3 / 2) t}\left[2+t-\frac{1+t}{\sqrt{(2-t) m+1}}\right]
$$

Therefore convergence is always uniform in this case.

Finally we consider pointwise convergence. The next result with $s=0$ is identical to Theorem 4.1 with $\beta_{i} \equiv 1$.

THEOREM 4.4. Suppose that $\sum a_{i} x^{i}$ is a power series such that the series $\sum\left(a_{2 i+t} /(2 i+t)^{s}\right)$ $(t=0,1)$ converge for some nonnegative number $s$, let $\gamma \geq 0$, and let $p_{n}(x)$ be ultraspherical with nonnegative constant $\rho \geq 2 s-1$. For every $j$ and $t$ the general Fourier coefficient $c_{2 j+t}$ of $\sum a_{i} x^{i}$ with respect to $\left\langle p_{n}\right\rangle$ exists and the following estimates hold.

(a) Assume that $\rho=2 s-1$. Then

$$
\left|c_{2 j+t}-\sum_{i=0}^{n} a_{i} m_{i, 2 j+t}\right| \leq L(2 j+t) \epsilon_{n}\left(\left\langle a_{2 i+t}(2 i+t)^{-s}\right\rangle\right),
$$


16 Least squares approximations of power series

where $L(2 j+t)$ is bounded as in (3.7). Moreover, if $n \geq 2|x|^{2 / s} /\left(1-|x|^{2 / s}\right)(:=0$ when $s=0)$, then

$$
\begin{aligned}
\left|\left[\sum a_{i} x^{i}-\sum_{j=0}^{n} c_{j} p_{j}(x)\right]\left(1-x^{2}\right)^{\gamma}\right| \\
\leq \sum_{t=0}^{1}\left[2(n+1)^{s}|x|^{(n+1) /(\gamma+1)} \lambda(n+1, \gamma)\right. \\
\left.\quad+\sum_{2 j+t \leq n} L(2 j+t) \mu(2 j+t, \gamma)\right] \epsilon_{n}\left(\left\langle a_{2 i+t}(2 i+t)^{-s}\right\rangle\right),
\end{aligned}
$$

where $\sum_{2 j+t \leq n} L(2 j+t) \mu(2 j+t, \gamma)$ is bounded by a polynomial in $n$ with degree term $\left(1 \cdot 3 \cdots\left(2 s^{\prime}-1\right) /\left(\rho^{\prime}+1\right) !\right) n^{\rho^{\prime}+1}$ if $\rho \neq 0$, and degree term $2 n$ if $\rho=0$. In particular, if

$$
\lim _{n \rightarrow \infty} n^{\rho^{\prime}+1} \epsilon_{n}\left(\left\langle a_{2 i+t}(2 i+t)^{-s}\right\rangle\right)=0 \quad(t=0,1),
$$

then $\sum_{j=0}^{n} c_{j} p_{j}(x)\left(1-x^{2}\right)^{\gamma}$ converges pointwise to $\sum a_{i} x^{i}\left(1-x^{2}\right)^{\gamma}$ on $(-1,1)$.

(b) Assume next that $\rho>2 s-1$. Then for each $j$,

$$
\begin{aligned}
\left|c_{2 j+t}-\sum_{i=0}^{n} a_{i} m_{i, 2 j+t}\right| & \leq 2 \max \left\{I(2 j+t)^{s},(n+1)^{s}\right\} m_{\max \{I(2 j+t), n+1\}, 2 j+t} \epsilon_{n}\left(\left\langle a_{2 i+t}(2 i+t)^{-s}\right\rangle\right) \\
& =O\left((n+1)^{-[\rho-(2 s-1)-r] / 2}\right) \epsilon_{n}\left(\left\langle a_{2 i+t}(2 i+t)^{-s}\right\rangle\right)
\end{aligned}
$$

for any $r$ in $(0, \rho-(2 s-1))$. And if $n \geq 2|x|^{2 / s} /\left(1-|x|^{2 / s}\right)(:=0$ when $s=0)$, then

$$
\begin{aligned}
& \left|\left[\sum a_{i} x^{i}-\sum_{j=0}^{n} c_{j} p_{j}(x)\right]\left(1-x^{2}\right)^{\gamma}\right| \\
& \leq \sum_{t=0}^{1} 2\left\{(n+1)^{s}\left[|x|^{(n+1) /(\gamma+1)} \lambda(n+1, \gamma)+\sum_{2 j+t \leq \rho_{n}^{*}} m_{n+1,2 j+t} \mu(2 j+t, \gamma)\right]\right. \\
& \left.\quad+\sum_{\rho_{n}^{*}<2 j+t \leq n} I(2 j+t)^{s} m_{I(2 j+t), 2 j+t} \mu(2 j+t, \gamma)\right\} \epsilon_{n}\left(\left\langle a_{2 i+t}(2 i+t)^{-s}\right\rangle\right),
\end{aligned}
$$

where $2 j+t \leq \rho_{n}^{*}$ if and only if $I(2 j+t) \leq n+1$; thus, $\rho_{n}^{*}$ may be solved from the quadratic inequality (c)(iii) in $j$ of Theorem 3.2. Furthermore,

$$
\sum_{2 j+t \leq \rho_{n}^{*}} m_{n+1,2 j+t} \mu(2 j+t, \gamma) \leq 1 .
$$


Proof. Inequalities (4.19) and (4.22) follow from Proposition 2.1 and Theorem 3.2 since

$$
\left|c_{2 j+t}-\sum_{i=0}^{n} a_{i} m_{i, 2 j+t}\right|=\left|\sum_{2 i+t>n} \frac{a_{2 i+t}}{(2 i+t)^{s}}\left((2 i+t)^{s} m_{2 i+t, 2 j+t}\right)\right| .
$$

Moreover, by (2.10) and (2.20),

$$
\begin{aligned}
\left|\left[\sum a_{i} x^{i}-\sum_{j=0}^{n} c_{j} p_{j}(x)\right]\left(1-x^{2}\right)^{\gamma}\right| \leq & \sum_{t=0}^{1}|x|^{t}\left|\sum_{2 i+t>n} \frac{a_{2 i+t}}{(2 i+t)^{s}}(2 i+t)^{s} x^{2 i}\left(1-x^{2}\right)^{\gamma}\right| \\
& +\sum_{t=0}^{1} \sum_{2 j+t \leq n}\left|\sum_{2 i+t>n} a_{2 i+t} m_{2 i+t, 2 j+t}\right| \mu(2 j+t, \gamma) .
\end{aligned}
$$

Therefore (4.20) and (4.23) follow from Proposition 2.1, (4.19), and (4.22), since for fixed $x$ in $(-1,1)$ and $t$, the sequence $(2 i+t)^{s} x^{2 i}\left(1-x^{2}\right)^{\gamma}$ is decreasing for $2 i+t>2|x|^{2 / s} /$ $\left(1-|x|^{2 / s}\right)$, and $\lim _{n \rightarrow \infty}(n+1)^{s}|x|^{(n+1) /(\gamma+1)}=0$ by the ratio test.

Suppose that $\rho=2 s-1$. Then by (3.7),

$$
\sum_{2 j+t \leq n} L(2 j+t) \mu(2 j+t, \gamma) \leq \sum_{j=0}^{\lfloor(n-t) / 2\rfloor} L(2 j+t)
$$

which is bounded by $2(\lfloor(n-t) / 2\rfloor+1)$ if $\rho=0$, and is otherwise bounded by

$$
\begin{gathered}
\frac{1 \cdot 3 \cdots\left(2 s^{\prime}-1\right)}{\rho^{\prime} !} \sum_{j=0}^{\lfloor(n-t) / 2\rfloor}\left(4 j+2 t+\rho^{\prime}\right)(2 j+t+1) \cdots\left(2 j+t+\rho^{\prime}-1\right) \\
=\frac{1 \cdot 3 \cdots\left(2 s^{\prime}-1\right)}{\rho^{\prime} !} 2^{\rho^{\prime}+1}\left[\frac{n^{\rho^{\prime}+1}}{2^{\rho^{\prime}+1}\left(\rho^{\prime}+1\right)}+\text { lower terms }\right] .
\end{gathered}
$$

(This follows from the observation that for any nonnegative integer $k, \sum_{i=1}^{n} i^{k}$ is a polynomial in $n$ with degree term $n^{k+1} /(k+1)$.)

Remark 4.5. $I(2 j+t)$ may be difficult to approximate in (4.23) when $s$ is not an integer (see Example 4.9(c)). If $s^{*}:=\min \{-\lfloor-s\rfloor,(\rho+1) / 2\}(\geq s)$ and $\sum\left(a_{2 i+t} /(2 i+t)^{s}\right)(t=0,1)$ converge, then so do

$$
\begin{gathered}
\sum \frac{a_{2 i+t}}{(2 i+t)^{s^{*}}}=\sum \frac{a_{2 i+t}}{(2 i+t)^{s}} \cdot \frac{1}{(2 i+t)^{s^{*}-s}} \\
\epsilon_{n}\left(\left\langle a_{2 i+t}(2 i+t)^{-s^{*}}\right\rangle\right) \leq \frac{2}{(n+1)^{s^{*}-s}} \epsilon_{n}\left(\left\langle a_{2 i+t}(2 i+t)^{-s}\right\rangle\right)
\end{gathered}
$$

by Proposition 2.1. Thus, although the resulting estimates with $s^{*}$ (instead of $s$ ) would be less accurate, we may use (4.20) when $s^{*}=(\rho+1) / 2$ and (4.23) with Proposition 3.3 when $s^{*}=-\lfloor-s\rfloor$. 
Example 4.6. Let $s=\rho=1$. Then $L(j)=2 j+1$ by (3.7), $\mu(2 j+t, \gamma) \leq 1$, and in (4.20) for $n \geq 2 x^{2} /\left(1-x^{2}\right)$, it follows that

$$
\sum_{2 j+t \leq n} L(2 j+t) \mu(2 j+t, \gamma) \leq\left(\left\lfloor\frac{n-t}{2}\right\rfloor+1\right)\left(2\left\lfloor\frac{n-t}{2}\right\rfloor+2 t+1\right)
$$

and thus we have pointwise convergence when

$$
\lim _{n \rightarrow \infty} n^{2} \epsilon_{n}\left(\left\langle a_{2 i+t}(2 i+t)^{-1}\right\rangle\right)=0, \quad t=0,1 .
$$

If $\rho / 4 \leq \gamma$, then by Lemma 2.2,

$$
\sum_{2 j+t \leq n} L(2 j+t) \mu(2 j+t, \gamma) \leq c\left(\left\lfloor\frac{n-t}{2}\right\rfloor+1\right)
$$

so pointwise convergence follows in this case when

$$
\lim _{n \rightarrow \infty} n \epsilon_{n}\left(\left\langle a_{2 i+t}(2 i+t)^{-1}\right\rangle\right)=0, \quad t=0,1 .
$$

Example 4.7. Let $s=1$ and $\rho=2$ in Theorem 4.4. Then $I(0)=2$, and by Proposition 3.3, $I(j)=(j+1)^{2}$ for $j \geq 1$. Therefore, since $\mu(2 j+t, \gamma)$ and $m_{I(2 j+t), 2 j+t}$ are bounded by one; if $n \geq 2 x^{2} /\left(1-x^{2}\right)$, then in (4.23),

$$
\begin{aligned}
& \sum_{\rho_{n}^{*}<2 j+t \leq n} I(2 j+t) m_{I(2 j+t), 2 j+t} \mu(2 j+t, \gamma) \\
& \quad \leq\left(\frac{t+1}{3}\right)\left(\left\lfloor\frac{n-t}{2}\right\rfloor+1\right)\left(2\left\lfloor\frac{n-t}{2}\right\rfloor+3\right)\left[(2-t)\left\lfloor\frac{n-t}{2}\right\rfloor+t+1\right] ;
\end{aligned}
$$

so we have pointwise convergence when

$$
\lim _{n \rightarrow \infty} n^{3} \epsilon_{n}\left(\left\langle a_{2 i+t}(2 i+t)^{-1}\right\rangle\right)=0, \quad t=0,1 .
$$

However, if $\rho / 4 \leq \gamma$, then by Lemma 2.2,

$$
\sum_{\rho_{n}^{*}<2 j+t \leq n} I(2 j+t) m_{I(2 j+t), 2 j+t} \mu(2 j+t, \gamma) \leq c \sum_{\rho_{n}^{*}<2 j+t \leq n}(2 j+t+1) ;
$$

so as in Example 4.6 pointwise convergence follows in this case when

$$
\lim _{n \rightarrow \infty} n^{2} \epsilon_{n}\left(\left\langle a_{2 i+t}(2 i+t)^{-1}\right\rangle\right)=0, \quad t=0,1 .
$$

Example 4.8. Let $s=2$ and $\rho=5$ in Theorem 4.4. By Proposition 3.3, $I(j)<R(j)+1$ for all $j$, and $R(j)=(j(j+5)+6) / 2<I(j)+1$ for $j \geq 3$. It follows that $I(j)=R(j)$ when $j \geq 3$ since $R(j)$ is an integer in this case. Therefore for $n \geq \max \{14,2|x| /(1-|x|)\}$, we have in (4.23),

$$
\sum_{\rho_{n}^{*}<2 j+t \leq n} I(2 j+t)^{2} m_{I(2 j+t), 2 j+t} \mu(2 j+t, \gamma) \leq \frac{1}{4} \sum_{\rho_{n}^{*}<2 j+t \leq n}[(2 j+t)(2 j+t+5)+6]^{2},
$$


where the latter sum is a polynomial with degree term $n^{5} / 10$. If $\rho / 4 \leq \gamma$ in this case, then by Lemma 2.2 the corresponding polynomial estimate is of degree four.

Example 4.9. Let us consider nonuniform approximation with Chebyshev polynomials, that is, $\rho=0 \geq 2 s-1$ in Theorem 4.4 .

(a) Let $s=1 / 2$. By (3.7), $L(j) \leq 2$, and thus if $n \geq 2 x^{4} /\left(1-x^{4}\right)$, then in (4.20),

$$
\sum_{2 j+t \leq n} L(2 j+t) \mu(2 j+t, \gamma) \leq 2\left(\left\lfloor\frac{n-t}{2}\right\rfloor+1\right) ;
$$

so we have pointwise convergence if

$$
\lim _{n \rightarrow \infty} n \epsilon_{n}\left(\left\langle a_{2 i+t}(2 i+t)^{-1 / 2}\right\rangle\right)=0, \quad t=0,1 .
$$

(b) Let $s=0$. By Proposition 3.3, $I(j)=j^{2}-2$ and in (4.23),

$$
\sum_{\rho_{n}^{*}<2 j+t \leq n} m_{I(2 j+t), 2 j+t} \mu(2 j+t, \gamma) \leq n-\sqrt{n+3} .
$$

(c) Let $0<s<1 / 2$. By (c)(iii) of Theorem 3.2, $i \geq I(j)$ if and only if

$$
j^{2} i^{s} \leq(i+2)^{2} i^{s}-(i+2)^{1+s}(i+1)
$$

(which may be used to find $\rho_{n}^{*}$ but can only be solved numerically for $i$, with $j$ fixed, to approximate $I(j))$. However we may obtain estimates to $(4.23)$ by replacing $s$ with $s^{*}=1 / 2$ and using (4.20) as in (a) above and Remark 4.5.

\section{Acknowledgment}

The author is grateful to the referee for suggesting several improvements.

\section{References}

[1] O. Aberth, Precise Numerical Analysis, Wm. C. Brown, Iowa, 1988.

[2] T. M. Apostol, Calculus. Vol. I: One-Variable Calculus, with An Introduction to Linear Algebra, 2nd ed., Blaisdell, Massachusetts, 1967.

[3] K. E. Atkinson, An Introduction to Numerical Analysis, 2nd ed., John Wiley \& Sons, New York, 1989.

[4] R. G. Bartle, The Elements of Real Analysis, 2nd ed., John Wiley \& Sons, New York, 1976.

[5] K. R. Davidson and A. P. Donsig, Real Analysis with Real Applications, Prentice Hall, New Jersey, 2002.

[6] J. Guyker, Legendre expansions of power series, The Rocky Mountain Journal of Mathematics 29 (1999), no. 4, 1271-1284.

[7] D. Jackson, The Theory of Approximation, American Mathematical Society Colloquium Publications, vol. 11, American Mathematical Society, Rhode Island, 1930.

[8] U. Luther and G. Mastroianni, Fourier projections in weighted $L^{\infty}$-spaces, Problems and Methods in Mathematical Physics (Chemnitz, 1999), Oper. Theory Adv. Appl., vol. 121, Birkhäuser, Basel, 2001, pp. 327-351.

[9] J. M. H. Olmsted, Advanced Calculus, The Appleton-Century Mathematics Series, AppletonCentury-Crofts, New York, 1961. 


\section{Least squares approximations of power series}

[10] E. D. Rainville, Special Functions, Macmillan, New York, 1960.

[11] T. J. Rivlin, The Chebyshev Polynomials, John Wiley \& Sons, New York, 1974.

[12] G. Szegö, Orthogonal Polynomials, American Mathematical Society Colloquium Publications, vol. 23, American Mathematical Society, New York, 1939.

James Guyker: Department of Mathematics, Buffalo State College (SUNY), 1300 Elmwood Avenue, Buffalo, NY 14222-1095, USA

E-mail address: guykerj@buffalostate.edu 


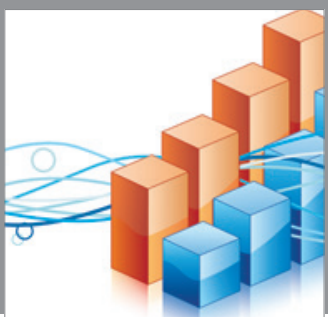

Advances in

Operations Research

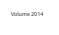

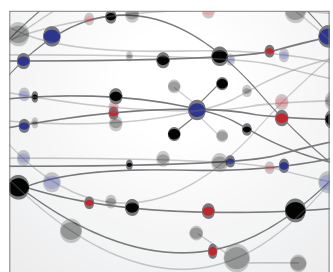

\section{The Scientific} World Journal
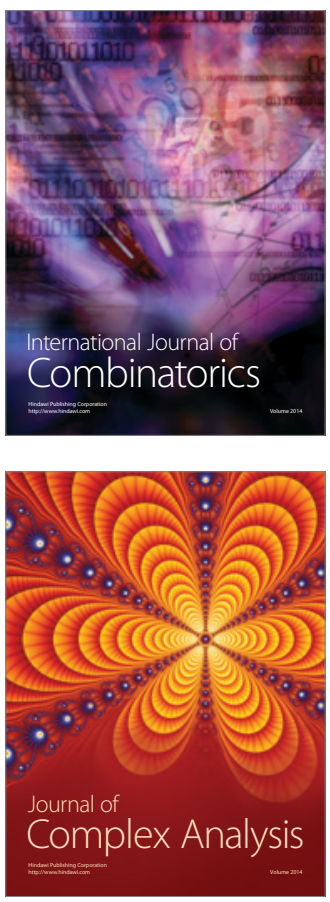

International Journal of

Mathematics and

Mathematical

Sciences
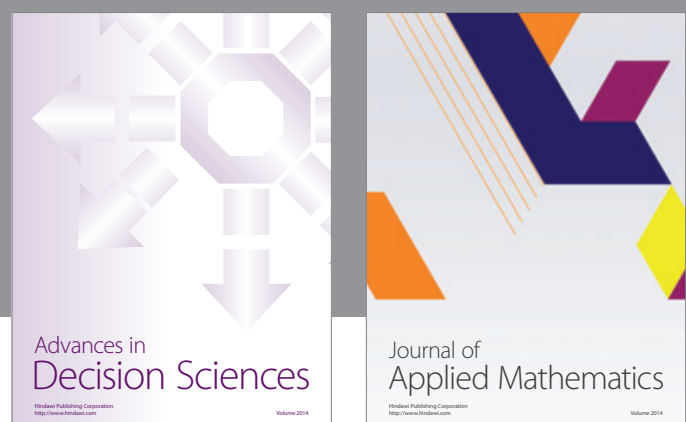

Journal of

Applied Mathematics
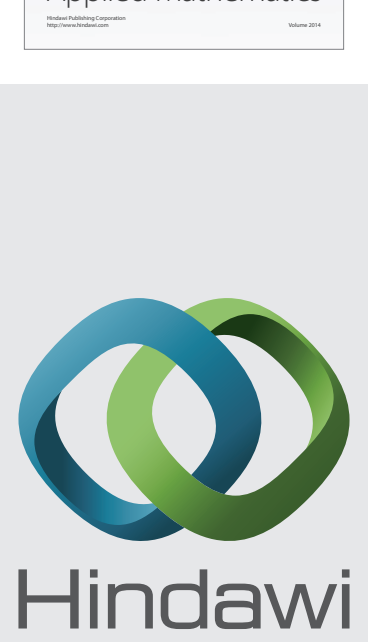

Submit your manuscripts at http://www.hindawi.com
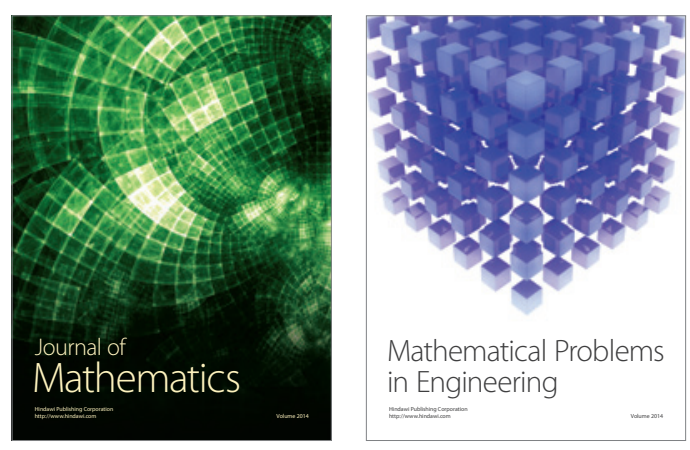

Mathematical Problems in Engineering
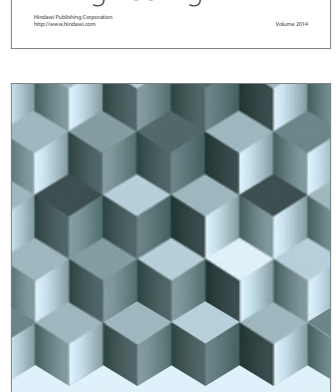

Journal of

Function Spaces
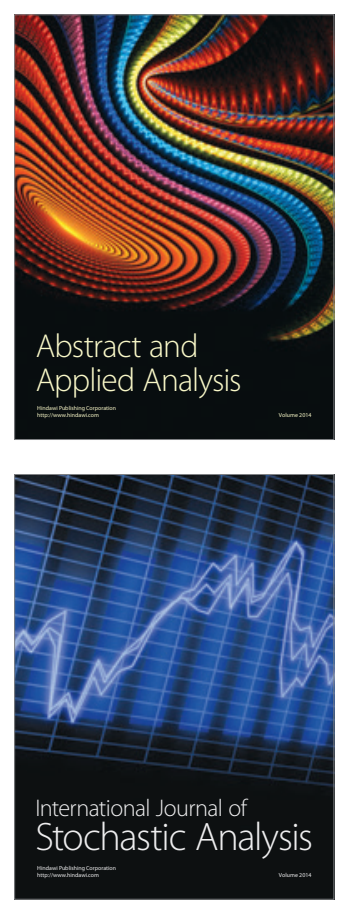

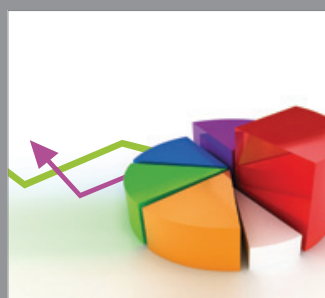

ournal of

Probability and Statistics

Promensencen
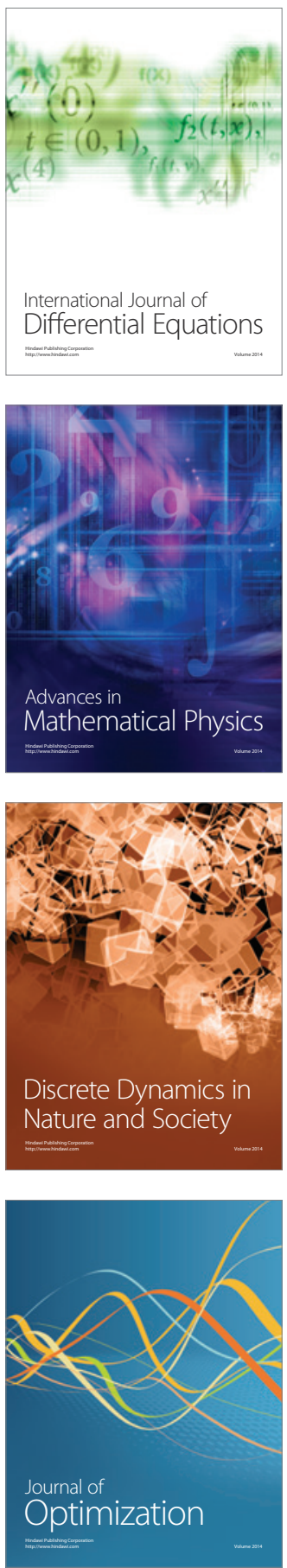